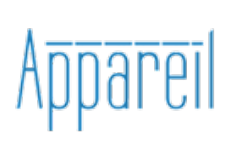

Appareil

$9 \mid 2012$

Penser l'art, penser l'histoire

\title{
Le goût baroque comme détermination d'un style : Wölfflin, Deleuze
}

\section{Anne Sauvagnargues}

\section{(2) OpenEdition}

1 Journals

Édition électronique

URL : http://journals.openedition.org/appareil/1413

DOI : 10.4000/appareil. 1413

ISSN : 2101-0714

Éditeur

MSH Paris Nord

Référence électronique

Anne Sauvagnargues, "Le goût baroque comme détermination d'un style : Wölfflin, Deleuze », Appareil [En ligne], 9 | 2012, mis en ligne le 02 juillet 2012, consulté le 30 juillet 2020. URL : http:// journals.openedition.org/appareil/1413; DOI : https://doi.org/10.4000/appareil.1413

Ce document a été généré automatiquement le 30 juillet 2020.

\section{(c) (i) () $९$}

Appareil est mis à disposition selon les termes de la Licence Creative Commons Attribution - Pas d'Utilisation Commerciale - Pas de Modification 4.0 International. 


\title{
Le goût baroque comme détermination d'un style : Wölfflin, Deleuze
}

\author{
Anne Sauvagnargues
}

1 La question de l'invention du goût - si importante pour la philosophie actuelle - exige un éclairage oblique, latéral, pour interroger la manière dont l'art devient un problème pour la philosophie, ce qui n'est pas possible sur le terrain de la philosophie seule. L'invention du goût, la posture de l'évaluation esthétique ne sont nées ni par génération spontanée, ni par nécessité interne : elles ont été préparées par un travail d'évaluation et d'élaboration théorique, sans doute, mais qui a été le fait des praticiens, puis des théoriciens de l'art (connaisseurs, historiens) avant de se proposer à l'attention des philosophes. Il y a donc une genèse historique du champ de l'art, qui comporte une lente constitution, une invention (au sens technique) des "agents", dirait Bourdieu, des acteurs ou des personnages, parmi lesquels les œuvres ellesmêmes; ceux qui les produisent, les philosophes qui les commentent ne sont pas les seuls en cause, ou plutôt n'apparaissent comme personnages principaux que dans un champ de réception qui comprend commanditaires et mécènes, mais aussi critiques, connaisseurs et goûteurs qui rendent leur existence possible ${ }^{1}$. Il ne s'agit pas pour autant de réduire l'apparition de l'esthétique à une sociologie de la constitution des biens culturels, mais de faire porter la philosophie sur l'individuation des objets d'art ${ }^{2}$, effet d'un procès qu'il faut évaluer pour conceptualiser l'art. L'émergence du problème du goût fonctionnant comme révélateur du statut de l'art pour la philosophie, le cas du baroque est ici exemplaire.

2 Si le baroque désigne aujourd'hui un certain répertoire d'œuvres dans la tranche historique qui, de la fin de la Renaissance au néoclassicisme, couvre le champ d'action de la production d'art européenne, l'étiquette elle-même cristallise tout un travail, qui montre à l'évidence que l'esthétique, qui prétend philosopher sur l'art, doit se faire épistémologie de l'histoire de l'art, pour prendre en compte son caractère nécessairement rétrospectif et les enjeux pragmatiques de sa constitution. C'est 
justement un tel complexe qu'il s'agit d'observer à l'œuvre dans l'histoire du baroque. En termes foucaldiens, l'apparition du baroque comme critère stylistique rend perceptible les œuvres en les unifiant ${ }^{3}$.

Il y a là un problème épistémologique qui s'inscrit en histoire de l'art de manière plus contraignante qu'en histoire générale car il lui est impossible de prétendre dissocier l'enquête historiographique d'un jugement normatif, l'objet étant " goûté » autant qu'il est expliqué par l'historien d'art qui le sélectionne ${ }^{4}$. Ce qui permet cette ubiquité, c'est la contemporanéité des œuvres, leur matérialité ${ }^{5}$, jointe à la perspective sous laquelle elles nous apparaissent comme témoins (monuments) d'un art qui nous touche peutêtre historiquement, mais dont l'effet n'est pas réductible à leur simple inscription historique ${ }^{6}$. S'agissant des arts plastiques que nous allons ici considérer, ce sont les œuvres elles-mêmes qui subsistent, qui se transforment dans leur monumentalité matérielle autant que dans l'idéalité de leur statut, telle peinture, architecture ou sculpture, s'étant lentement individuée pour correspondre au concept d'«art baroque ", à mesure qu'une telle notion prenait consistance en histoire de l'art.

Les œuvres sont donc indissociables du complexe qui les rend sensibles. Il comprend, à côté de leurs propriétés intrinsèques (leur texture matérielle et formelle autant que leur détermination locale, leur situation spatio-temporelle sur laquelle s'appuie l'historiographie de l'art), le travail sur le goût qui s'est opéré à travers elles, qui les fait entrer dans le répertoire d'une histoire de l'art occidentale, qui alimente notre définition de l'art. L'invention du goût baroque exige de revenir sur les conditions épistémologiques de son institution historiographique, qui met en œuvre une philosophie de l'art et de l'histoire. Chez Quatremère de Quincy, «baroque » n'a qu'un sens péjoratif; avec Jakob Burckhardt, il prend un sens descriptif encore largement dévalorisant. Il faut attendre Wölfflin, l'élève de Burckhardt, pour que l'étiquette "baroque ", d'épithète dépréciatrice, prenne le sens descriptif que nous lui connaissons aujourd'hui. Mais d'autre part, si on considère le répertoire des œuvres nommées aujourd'hui "baroques", on voit bien qu'une alternative entre détermination sociologique externe (l'art baroque, résultat de notre détermination actuelle de l'art) et essentialisme (le baroque comme essence supra-temporelle) est méthodologiquement ruineuse, trop large, inopérante ${ }^{7}$. C'est pourquoi Deleuze les rejette ensemble en montrant qu'il s'agit de penser ensemble la perspective sous laquelle l'esthétique théorique, la production d'œuvres déterminées et leur institution sociale interagissent. C'est à cela que répondent le concept deleuzien de « maniérisme » et sa théorie de la création culturelle comme « art mineur ».

\section{L'invention du baroque : goût et histoire}

5 Ce que nous unifions rétrospectivement sous l'étiquette "baroque " ne s'est pas d'abord signalé à l'appréciation contemporaine comme l'émergence d'un style rompant avec la continuité classique ${ }^{8}$ de la norme renaissante. Les œuvres effectivement réalisées et conservées, auxquelles on prête aujourd'hui une valeur de rupture, passaient inaperçues, semblaient inconsistantes ou forçaient l'attention. Rien ne permettait de les faire entrer dans une catégorie unique. Ce qui est plus décisif encore, rien ne permettait à l'époque de les considérer comme l'émergence d'une norme alternative au classicisme renaissant. L'émergence d'une catégorie «baroque » naît du croisement entre l'éclairage néoclassique, favorisant une théorie de l'imitation comme 
conformation au modèle (héritée de la Renaissance, active au XvII ${ }^{\mathrm{e}}$ siècle chez Bellori ${ }^{9}$, prégnante au XviII ${ }^{\mathrm{e}}$ siècle, par exemple chez Winckelmann, et manifeste au début du XIX ${ }^{\mathrm{e}}$ siècle chez Quatremère de Quincy), et la théorie du génie et d'une production de règles dans la culture, dont la Critique de la faculté de juger de Kant se fait l'écho en 1790. Ce sont les historiens d'art qui mettent en forme le statut de l'art pour la philosophie, et permettent d'observer comment un style post-renaissant, qui n'a pas reçu encore de dénomination particulière, prend cette identité rétrospective et négative de «bizarrerie hors norme » avant de recevoir, avec Wölfflin, ses lettres de noblesses en 1888 , et de devenir un style ${ }^{10}$ à part entière, c'est-à-dire un répertoire de formes identifiables et susceptibles d'être décrites, et non une déviation. Il faut attendre en effet la publication du magistral Renaissance et baroque pour que le statut du baroque glisse de l'anormal, de la dégénérescence, vers l'idée d'un style autonome. C'est à ce point seulement que "le baroque" se construit comme un problème pour la philosophie. Il n'y a d'œuvre «baroque » que sous cette perspective, et récapitulant ce procès. C'est pourquoi la constitution de la notion est exemplaire pour la manière dont l'objet d'art se prête à la réflexion philosophique par l'intermédiation de tout un procès du goût et de la critique d'art.

Il s'agit ici de réfléchir sur le travail de nomination qui préside à l'élaboration d'une étiquette descriptive en histoire de l'art - ce qui pose aussi bien le problème du statut d'une telle discipline. Le baroque devient lentement un style, au sens d'instrument méthodologique pour l'historien d'art, comprenant des caractéristiques morphologiques, techniques, spatio-temporelles qui permettent datation, attribution et classification, sous l'unité d'une norme qui est celle de la définition de l'art. Mais ce n'est pas tout. Le baroque ne montre pas seulement le travail de constitution d'une étiquette stylistique, il est exemplaire pour une raison plus forte. Pour cette culture européenne qui invente l'esthétique, qui scinde les arts de la sphère technique, l'âge baroque et les œuvres qu'il produit se signale comme cette période, cette mutation qui achève la conquête renaissante, borde la période, la clôt. Cela rend son examen pertinent pour comprendre les mutations du goût, solidaires de la constitution de l'esthétique philosophique. La succession historique, cette postériorité chronologique, d'abord réfléchie par les théoriciens du temps sur le mode d'un équilibre délicat, un heureux hasard (c'est le statut de Michel-Ange pour Vasari) puis sur le mode de la dégénérescence ${ }^{11}$, signale le baroque comme exemplaire pour le statut même de l'histoire de l'art.

7 Pour le père de l'histoire de l'art, Vasari, artiste lui-même, Michel-Ange - que Wölfflin présente sans façon comme «le père du baroque ${ }^{12}{ }^{2}$-, incarne la norme classique triomphante, c'est-à-dire la perfection antique et la nature de l'art. À la limite, il n'y a plus rien à espérer de l'art après le point de perfection que représente Michel-Ange ${ }^{13}$. C'est que Vasari se fait du style (au double sens de répertoire morphologique et de réussite exemplaire) une conception binaire, normée par l'idéal antique. Il n'y a qu'une seule buona maniera, la manière antique, assimilée par lui à une nature de l'art, tout simplement incompatible avec toute autre exécution: la maniera greca (grecque, byzantine) ou la maniera tedesca (tudesque, gothique) du passé, ou les échecs contemporains. La norme de l'art est présente ou absente, et il n'y a aucun lieu de définir les répertoires hors norme, qui indiquent seulement des lacunes de l'histoire, des catastrophes pour la civilisation ${ }^{14}$. Le concept même de baroque, d'un art alternatif à la norme classique, n'a aucun sens pour Vasari. Cela ne l'empêche pas de goûter 
précisément chez Michel-Ange - comme Wölfflin, lecteur de Kant ne manque pas de le remarquer -, ces marques qui en font un génie puissant, original, capable de détourner les règles, de se soustraire à l'imitation servile pour produire un usage exemplaire de la norme ${ }^{15}$.

Les artistes ont à son égard une dette infinie et perpétuelle, car il a rompu les liens et les chaînes qui les faisaient travailler tous continuellement de la même manière ${ }^{16}$.

N'en concluons pas que Vasari ait pu, au milieu du XvI siècle, se montrer sensible à une variation baroque qui n'existait pas. C'est plutôt que Vasari, artiste lui-même, successeur de Michel-Ange, ne dispose pas de critère pour penser le changement en histoire et réfléchir sa propre situation d'historiographe, c'est-à-dire de témoin soucieux de recollecter une longue portion d'œuvres du passé.

C'est avec Bellori au XvII siècle, puis avec Winckelmann ${ }^{17}$ un siècle plus tard, que la question des changements culturels est thématisée sous le schème vital du vieillissement (Vasari ne dépassant pas l'âge de la maturité), en même temps que l'idéal de l'art est réaffirmé avec force comme un canon constant. L'âge baroque reçoit de ce fait l'identité suspecte d'une variation de la norme classique, que l'on ne peut entendre que comme une dégénérescence, faute de pouvoir envisager une historicité du canon. Cela révèle une théorie implicite de l'histoire qui commande le travail du goût, et l'appréciation, par l'Europe cultivée, de son propre tribut à la civilisation des arts. La période du Seicento pose à l'esthétique naissante le problème du vieillissement dans l'histoire, d'une dégénérescence sénile des civilisations - tandis que l'origine antique, cessant d'être présente comme nature, est prise en vue nostalgiquement dans la longue perspective temporelle qui sépare l'Europe de l'heureuse Grèce. C'est particulièrement net dans l'œuvre de Winckelmann, l'archéologue qui exhume pour l'Europe les témoins subsistant de l'art grec, les présentant en même temps comme la nature de l'art, et la ruine de l'histoire, tout en stigmatisant l'art de son temps ${ }^{18}$. La nature de l'art s'expose historiquement pour l'Europe comme une origine perdue (la Grèce classique), tandis que les œuvres du présent, jouant avec le répertoire classique, montrent que la référence à l'Antiquité cesse de s'imposer comme évidence atemporelle. C'est bien la nécessaire historicité de cette référence à l'antique qui s'impose à l'Europe. Lorsque les œuvres de la fin de la Renaissance sont réfléchies dans le discours sur l'art comme ces œuvres qui font varier la norme, elles reçoivent le statut d'un art finissant, art tardif, et sont indexées sur une théorie sociopolitique de la décadence des mœurs autant que du goût. C'est particulièrement net dans la constitution de l'étiquette "baroque » et révèle, par-delà le divorce entre production réelle et définition intellectuelle de l'art qui continue à travailler l'âge moderne, la fonction opératoire de l'art à l'âge de l'esthétique : il contribue à la théorie de l'histoire et vaut comme symptôme de la santé politique des civilisations.

\section{L'anomalie baroque : la tératologie du goût}

10 Rappelons rapidement le travail de cristallisation qui a produit l'étiquette baroque. Comme tous les commentateurs ${ }^{19}$ n'ont pas manqué de le rappeler, l'appellation «baroque » n'a pour les contemporains aucun sens, pas même péjoratif, puisque le mot vient du portugais barroco, qui désigne au $\mathrm{XvI}^{\mathrm{e}}$ siècle une perle irrégulière ${ }^{20}$. C'est dans cette acception que Furetière l'accueille en 1690 dans son Dictionnaire universel ${ }^{21}$ et qu'il 
est repris par le Dictionnaire de l'Académie dans sa première édition de $1694^{22}$. Il faut attendre l'édition de 1740 du Dictionnaire de l'Académie pour voir apparaître l'extension d'un sens figurée ${ }^{23}$ :

Baroque se dit aussi au figuré pour irrégulier, bizarre, inégal. Un esprit baroque, une expression baroque ${ }^{24}$.

De l'extension figurée, le terme fait donc son apparition en histoire de l'art par une évolution sémantique interne au français, et de là essaime dans le vocabulaire italien ${ }^{25}$ et allemand ${ }^{26}$. C'est au jeune Quatremère de Quincy que revient l'honneur d'attester l'emploi du terme pour l'histoire de l'architecture: il propose une définition du baroque architectural dans l'Encyclopédie méthodique (1788) qu'il reprend en 1832 dans son Dictionnaire de l'architecture ${ }^{27}$, non sans faire subir au terme une torsion dont il est lui-même inconscient. L'épithète "baroque " ne qualifie pas encore un style, mais employée comme adjectif qualificatif, elle stigmatise une dénaturation du goût, et donc est prise au sens figuré de goût baroque. Quatremère en repère néanmoins les exemples dans ce répertoire précis d'œuvres qui, à la suite de Wölfflin, recevra la dénomination stylistique et neutre de «baroque». C'est pourquoi sa définition marque le basculement du terme du langage courant vers l'histoire de l'art.

La mutation sémantique qui offre l'adjectif à la plume de Quatremère est elle-même exemplaire. Il n'est pas indifférent que «baroque » s'applique en joaillerie à des perles qui ne sont pas parfaitement rondes, à un affaiblissement du standard de la perle perfection géométrique autant que valeur marchande - qu'on transpose de l'objet précieux à l'esprit, puis au goût baroques. De la perle " parfaite » - normale - à l'ellipse ovoïde baroque, ce parcours signale un intérêt pour les anamorphoses de la forme. Qu'il s'agisse dans le vocabulaire technique de la joaillerie d'une dégradation du produit, d'une perte de valeur, indique le statut de cette transformation, comprise comme déviation de la norme, comme dénaturation. Ce qui est frappant dans l'usage que Quatremère fait du terme, c'est que "baroque » prend le sens d'un écart, d'une déviation, figure interprétée comme une translation du propre au figurées ${ }^{8}$. C'est en cela que le baroque pose de manière kantienne le problème de la production d'une nouvelle règle, d'une originalité d'abord incapable d'être reçue parce qu'elle choque le goût, d'une originalité qui n'est pas encore devenue exemplaire ${ }^{29}$. Comme le note Deleuze, "les perles irrégulières existent, mais le Baroque n'a aucune raison d'exister sans un concept qui forme cette raison même ${ }^{30}$ ». Cette raison est fournie par l'usage d'une norme invariante dont l'histoire de l'art a besoin pour constituer ses collections. L'art baroque apparaît comme une figure du classique non tant par ses propriétés intrinsèques (un goût baroque pour l'anamorphose et l'allégorie) que parce que la déformation même est interprétée comme translation. C'est une théorie du sens propre qui accompagne le parcours de visibilité du baroque, une théorie implicite de la norme invariante qui commande la migration du terme de la joaillerie vers histoire de l'art. D'où l'intérêt de la période baroque et du statut que reçoivent ses productions pour une philosophie comme celle de Deleuze, qui cherche à penser la norme dans les productions d'art comme variation et minoration (maniérisme).

Sous la plume de Quatremère, « baroque » n'est encore qu'un qualificatif dépréciatif et moral, par lequel on condamne un excès architectural, une "nuance du bizarre", bizarre "superlatif », qui «entraîne " l'idée de «ridicule poussé à l'excès ${ }^{31}$ ». Le baroque, présenté comme adjectif en tête de l'article, est chemin faisant substantivé, comme la surenchère d'un excès. "Superlatif» du bizarre, c'est à l'article 
correspondant (« BIZARRERIE »), que Quatremère livre sa définition du style baroque, et exécute Borromini, qui «a donné les plus grands modèles de bizarrerie ${ }^{32}$ ». Or, la bizarrerie est réfléchie par Quatremère comme une disposition passionnelle et non stylistique, qui fait l'objet d'une condamnation morale. Ce qui est frappant dans cette définition peu inventive, passionnante par sa confusion même, c'est qu'elle insiste sur une théorie de la dépravation subjective bien qu'elle l'explique par la dégénérescence historique. S'il existe des «exemples frappants » de ce goût baroque, si les productions d'art du siècle précédent livrent bien des échantillons durables de cette dépravation, ces échantillons (l'architecture de Borromini, le Saint-Suaire de Turin de Guarini) sont réductibles au dérèglement passionnel, à la dépravation du goût subjectif, au vice personnel. Ce vice a une double cause, qui forme les «deux principes généraux » qui chez les modernes «surtout" "produisent la bizarrerie dans le règne des arts ». Le premier est politique et concerne "la nature ou la constitution des nations modernes »; le second, éthique, et passionnel, dépend " en particulier des passions et des intérêts qui exercent leur empire sur les artistes ${ }^{33}$ ». C'est la perfection de l'art qui " en amène la chute », dans le pays même où le goût avait atteint sa plénitude la plus manifeste: «L'Italie moderne nous offre, dans les révolutions que l'architecture a subies chez elle, la preuve la plus frappante de ce qu'on vient d'avancer » : « les chefsd'œuvre s'y sont multipliés ", n'est-ce pas ce qui devait «maintenir le goût dans sa pureté, et le préserver au moins des grands écarts? Cependant le siècle suivant devait être celui de la bizarrerie».

La perfection rapide de l'art devait, dit-on, en amener la chute. Le génie parut épuisé par les efforts qu'il avait faits, les yeux s'étaient lassés des formes simples: on appela la simplicité monotonie, la sagesse froideur : l'attachement aux règles parut de la stérilité, et l'innovation prit la place de l'invention. On sait assez ce que furent les ouvrages produits sous l'influence de ce principe malfaisant (Voyez

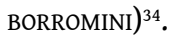

14 Le principe de cette théorie qui affiche une nostalgie passéiste assez naïve, c'est l'invariance d'un modèle excellent, mais donné au passé, auquel Quatremère oppose la variabilité du goût, l'inconstance humaine qui remplace "l'invention" (véritable originalité) par "l'innovation ", source de tous les vices ${ }^{35}$. On isole ici très clairement la théorie d'une décadence des arts, pensée comme une corruption des mœurs - de l'artiste - qui relève d'une dégénérescence sociopolitique, par excès de civilisation. Winckelmann l'observait pour la production d'art en Grèce : le point d'apogée du grand style basculait par maturation interne dans la dégénérescence du style gracieux, puis du style d'imitation ${ }^{36}$. Chez Winckelmann, l'argument est stylistique : l'art de la Grèce hellénistique dégénérait par imitation maniérée des exemples classiques au lieu de se régler sur la nature de l'art, mais il l'appuie sur l'argument politique de la perte de la liberté en Grèce $^{37}$; chez Quatremère, la décadence suit peut-être la corruption sociopolitique, mais sa racine est uniquement passionnelle. Les périodes d'excellence sollicitent ce vice singulier qui détourne de l'imitation par amour de la gloire. C'est ce qui explique les innovations architecturales de Borromini.

Borromini ne fit donc autre chose que renverser tout le système de l'architecture grecque sans le remplacer par aucun autre ${ }^{38}$.

Borromini n'est pas coupable d'avoir renversé le système de l'architecture classique sans l'avoir remplacé par aucun autre que le sien, il est d'abord coupable d'avoir attenté au canon. C'est le fait d'avoir voulu se soustraire à l'imitation que Quatremère condamne. Le Bernin devrait faire l'objet d'une condamnation tout aussi vigoureuse, 
mais Quatremère le dédouane, parce qu'il peut rapporter le Baldaquin de la confession de Saint-Pierre ${ }^{39}$ (dont les colonnes torses sont tellement symptomatiques du baroque romain) à une citation par «tradition et imitation » de l'ancienne confession ${ }^{40}$. C'est bien la définition de l'art qui est en jeu: Quatremère ne peut tolérer que ces œuvres susceptibles d'être rapportées à un modèle ancien qu'elles reprennent. Le modèle n'est plus la nature, mais l'exemple culturel réifié par l'histoire. L'imitation de la tradition (et non comme chez Winckelmann, l'imitation de la nature), c'est-à-dire l'observation d'une norme culturellement donnée, détermine le partage entre art valide et art bizarre. Ce qui définit l'art, c'est l'observation d'une norme livrée par l'histoire de l'art et considérée comme intangible. Quatremère de Quincy introduit l'épithète "baroque " en histoire de l'art parce qu'il s'appuie sur cette définition de l'art comme imitation invariante.

C'est pourquoi la condamnation de l'art bizarre n'est pas stylistique (un art laid), mais morale : un art vicieux. La bizarrerie vicieuse admet comme degré inférieur, le caprice, son degré supérieur étant occupé, on l'a vu, par le superlatif baroque. Les maîtres du passé, Michel-Ange et Vignola, ont "quelques fois admis des détails capricieux », par « inconséquence » et "jeu puéril »; Borromini et Guarini sont « les maîtres du genre bizarre ». Le caprice n'est qu'une provocation de l'enfance (une immaturité) qui relève de "l'oubli des règles" (et d'une bonne correction!), même si ses suites peuvent « devenir dangereuses ", il peut « se montrer quelques fois sans la bizarrerie » - quand il s'agit de maîtres illustres. La bizarrerie n'a plus le caractère du jeu indiscipliné de l'enfance, c'est une maladie déclarée, qui peut se révéler "contagieuse ». Alors qu'il affirme que son principe est passionnel, Quatremère soutient qu'elle est bien moins contagieuse dans les mœurs que dans le goût.

On observera que le vice de la bizarrerie dans les mœurs est une maladie ordinairement de quelques individus, lorsque dans les arts on voit qu'elle devient, si l'on peut dire, une épidémie ${ }^{41}$.

Le vocabulaire de l'épidémie est révélateur : la bizarrerie baroque, maladie morale, se cantonne à la sphère des arts, qui payent leur récente élévation à la dignité d'une sphère autonome de la culture d'une pathologie spécifique. Le baroque signale une tératologie du goût, justiciable d'une condamnation morale, mais spécifique à l'art. Ce vice est contagieux - sans pour autant que sa force de propagation le valide comme mutation du goût. L'épidémie atteste pourtant la vigueur d'une nouvelle norme. Mais cette pluralité ne peut être admise par Quatremère, que ce divorce entre une morale du goût abstraite et son effectuation empirique ne gêne pas. Une telle détermination abstraite de l'art produit une théorie du mauvais goût, baroque, bizarre, hors normes, contre-nature. C'est dans ces conditions que l'épithète baroque est reçue en histoire de l'art, comme mauvais goût, qui résulte de l'excellence même de la culture. "Plus les chefs-d'œuvre se sont multipliés, plus il parait difficile d'en produire qui semblent nouveaux ${ }^{42}$ ». Le goût est corrompu par excès de culture, perversion en apparence symétrique de l'inculture (sauvagerie) et de la désobéissance (enfance), mais infiniment plus dangereuse, beaucoup plus condamnable, et inquiétante puisqu'elle manifeste le retour à la barbarie d'une culture énervée par sa perfection même ${ }^{43}$. L'étiquette baroque naît pour les arts dans ce contexte: un principe de condamnation de la nouveauté interprète la transgression du répertoire des formes réifiées par l'histoire comme vice individuel, déclenché par l'excellence de la culture passée. 
BIZARRERIE, s. f., terme qui exprime, dans l'architecture, un goût contraire aux principes reconnus, une recherche affectée de formes extraordinaires, et dont le seul mérite consiste dans la nouveauté même qui en fait le vice ${ }^{44}$. principes de l'art, le recours à la théorie passionnelle, au vice privé, vient masquer la contradiction. Borromini n'est pas un architecte cherchant à résoudre les problèmes constructifs et stylistiques hérités de la tradition. C'est un individu suspect, mû par un "faux amour de gloire et de distinction », un "faux système d'étude » et un "principe d'envie ». Quatremère lie la production en art à un principe subjectif transgressif, mais cette subjectivité qui rejette l'imitation servile ne prend pas chez lui la figure du " génie ", qui bouleverse les règles du goût en suivant la règle de la nature. Tout en conservant le même cadre, elle prend la figure de "l'ambitieux", qui confond «la singularité avec l'originalité ». Quatremère réduit la production subjective à l'originalité incapable de devenir exemplaire (Unsinn ${ }^{45}$. Quoiqu'il fasse de l'initiative individuelle le principe d'une transformation des œuvres, il ne dispose pas d'un cadre pour penser la production du nouveau dans la culture, contrairement à Kant, dont la théorie du génie permet clairement de théoriser les mutations du goût collectif. Privé de l'inspiration kantienne qui comprend l'exemplarité du génie comme obéissance à la règle de la nature et non comme l'observation des canons de la culture, Quatremère oppose une subjectivité déréglée à l'appareil des normes transmises par l'histoire de l'art, que la conscience historique réifie comme une nature de l'art tout en la projetant dans le passé historique. Il produit donc cette théorie désenchantée, où la chute des arts provient de l'incapacité individuelle à supporter l'excellence et la multiplication des chefs-d'œuvre. On comprend alors le lien de l'art baroque à l'histoire. Le baroque est produit par l'histoire, de l'avis de ses premiers théoriciens mêmes, c'est une maladie de la conscience antiquaire ${ }^{46}$.

19 Ces précisions permettent de mesurer la rupture épistémologique qu'introduit Wölfflin, et l'intérêt des productions de cette période comme de leur statut pour une théorie de la création et de la mutation des cultures.

\section{Anomie, formalisme et matériologie : Wölfflin, Deleuze}

Wölfflin reprend en apparence la théorie de la dégénérescence de l'art, par excès de civilisation, mais en se proposant d'étudier la «dissolution» (Auflösung) de la Renaissance, il considère l'art tardif comme un style à part entière.

On a pris l'habitude d'entendre sous le nom de baroque le style qui marque la dissolution de la Renaissance, ou - selon une expression plus fréquente -sa dégénérescence ${ }^{47}$.

Ce faisant, il inaugure pour l'histoire de l'art une nouvelle époque qui rompt décisivement avec la théorie de la dégénérescence. Wölfflin indique la position chronologique du baroque (la fin de la Renaissance) et la projette comme ses prédécesseurs sur le double axe politique et axiologique, en reprenant la théorie politique du déclin des civilisations, qui s'accompagne d'une théorie axiologique de la dégénérescence de la culture (des mœurs et du goût). Mais en faisant du baroque un style, Wölfflin ouvre un nouveau chapitre de la théorie des arts. L'unicité de la norme, l'invariance du canon antique sont remplacées par la constitution d'un style nouveau, 
qui marque un nouveau départ. C'est une rupture épistémologique. Il y a une pluralité des normes, une vie des normes, indépendante de la longévité des canons. La norme renaissante peut mourir, le baroque forme un nouveau style, et la production d'art n'est pas réductible à une norme invariante.

Une telle position oblige Wölfflin à proposer simultanément une critique de l'histoire de l'art par une refonte catégorielle et à proposer une matériologie du baroque. Il utilise d'abord le répertoire médical nietzschéen de la symptomatologie pour échapper au schème de la décadence.

La deuxième Renaissance ne se perd pas dans un art décadent, spécifiquement distinct, mais de l'apogée, le chemin mène directement au baroque. Toute innovation est un symptôme du style baroque naissant ${ }^{48}$.

Le baroque n'est plus un signe épidémiologique mais le symptôme d'une innovation, et Wölfflin se propose d'étudier ce " complexe de symptômes qui constitue le baroque ${ }^{49}$ ». L'âge baroque et sa production reçoivent de ce fait la consécration d'une transformation singulière et spécifique de la culture. De la tératologie du goût singulier, on passe à l'analyse de "l'essence de la transformation stylistique ». En considérant la production qui marque la dissolution de la Renaissance, Wölfflin se propose

de reconnaître, si c'est possible, dans la dénaturation (Verwilderung) et l'arbitraire

(Willkür) la loi qui permet de donner un aperçu sur la vie interne de l'art ${ }^{50}$.

Il prend ainsi le contre-pied de son maître, Burckhardt ${ }^{51}$, qui tenait le baroque pour une variante de la langue classique, retournée à l'état sauvage, et qui écrivait dans son fameux Cicerone :

L'architecture baroque parle la même langue que l'architecture classique, mais il

s'agit d'un dialecte ensauvagé (verwilderten) ${ }^{52}$.

Wölfflin répond: "le langage formel a changés3». Ce qu'on entendait comme dénaturation (perte collective) ou arbitraire (bizarrerie individuelle) constitue les symptômes d'un style différent, qui donne un aperçu sur la vie de l'art (au singulier), donc sur la succession des styles dans l'unité d'une tradition. La rupture tient à ceci que dans le cadre même où se situait Quatremère, et sous l'unité d'une seule tradition, Wölfflin envisage une pluralité, une évolution des styles. Le passage au registre de la langue marque une double rupture avec la théorie politique et morale précédente : la Verwilderung reprend l'idée de décadence, au sens d'un retour à la nature, qui implique moins l'idée d'une dépravation que celle d'une exténuation de la culture : elle reste le syndrome des civilisations qui meurent, conserve l'idée d'une dégénérescence sénile ${ }^{54}$. Mais le passage du schème des âges de la vie (dégénérescence sénile) à celui de la langue montre que le style baroque fait maintenant l'objet d'une étude morphologique spécifique.

Le baroque n'est plus considéré comme l'exténuation de la culture classique, mais comme une "nouvelle langue» avec ses principes formels propres ${ }^{55}$. Rapporter les principes stylistiques des arts plastiques à la sphère linguistique permet, mieux que l'idée de "civilisation» en général, à laquelle Burckhardt restait attachée, de déterminer la singularité de chaque style. Là où Burckhardt parle d'exténuation dialectale en utilisant le schème de la dénaturation, de l'affaiblissement d'une langue dénaturée par un usage qui la gauchit, Wölfflin invoque l'incomparable diversité des langues, leur complétude, leur différence. Il ne s'agit pas d'une variation dialectale, du gauchissement d'un usage normatif mais d'une autre langue, tout autant capable que la 
première de signifier, avec ses moyens propres. En fait, le passage au répertoire de la langue indiquait déjà chez Burckhardt cet infléchissement: quelle que soit leur nostalgie pour les langues classiques, les Européens ont depuis longtemps habilité leurs propres langues vernaculaires, et admis dans les faits la mutation des langues. Comprendre le style pictural comme une langue indépendante confirme Wölfflin dans son entreprise: déterminer les principes stylistiques propres au baroque et les déterminer sur le plan formel ${ }^{56}$, non que Wölfflin applique à la grammaire plastique du classique et du baroque des catégories linguistiques, mais il se sert de l'analogie avec la langue pour déterminer des paires d'oppositions contrastées qui permettent de faire apparaître le baroque comme un style nouveau, et non comme une variante exténuée du classique.

Or, ce recours à la langue est passionnant, si on le met en rapport avec le concept de «minorité ${ }^{57}$ » chez Deleuze, qui s'applique précisément au principe de la nouveauté stylistique à partir d'une analyse des variations linguistiques. On peut bien comprendre la langue, en dépit de ses variations continuelles, en la rapportant à une norme standard, en concevant la norme comme un holotype transcendant, comme l'invariant de ses transformations. Mais une telle position est incapable de prendre en compte le changement. Il faut alors concevoir la normativité comme une variabilité, la variation incessante d'un usage, à l'intérieur d'un certain seuil. De la même manière, l'art d'une époque se définit par une normativité, donnée pour l'Europe par la norme classique, dont le baroque constitue bien, comme le voulait Burckhardt, une variation dialectale. Mais cette variation montre plutôt la vitalité des arts. Si toute langue, ou toute définition des arts, peut à un moment donné exercer sur la culture du temps la domination d'un étalon de valeur (ce que Deleuze appelle l'usage majeur d'une langue ou d'une culture), la création des cultures, comme la vie des langues, s'explique par leur usage déviant, qui pousse la norme sur ses bords par un usage mineur, qui rend compte de la variation des normes, de la transformation des cultures, mais aussi de ce que toute production atypique devienne à son tour susceptible de fonder une nouvelle norme majeure. Majeur et mineur ne qualifient donc pas les normes elles-mêmes, mais seulement leur usage.

L'art de l'âge baroque n'est pas déviant en soi. Simplement, sa situation historique prédisposait cet art savant, se vivant lui-même comme l'héritier de la norme classique, à porter cet héritage jusqu'à son point de rupture, tout comme sa situation devait déterminer son statut pour l'histoire de l'art. Ceci explique pourquoi les étiquettes stylistiques sont créées a posteriori, et dans une acception le plus souvent péjorative ${ }^{58}$. On appelle baroque ce qui n'est pas conforme au canon classique, ce qui résiste à la norme, par trop de naturel (le Caravage ${ }^{59}$ ), ou trop de fantaisie (Borromini). La première unité du terme «baroque » est donc négative. En même temps, la production d'une étiquette négative et dévalorisante indique un moment de l'histoire du goût, où la norme qui discrimine bon et mauvais goût devient sinon perméable, du moins montre sa face interne. La bordure du mauvais goût est perceptible, identifiable, et dans la perspective de l'histoire de l'art déjà constituée, elle devient choquante. C'est sur le fond de cette productivité de la norme classique que l'élision des œuvres baroques oblige l'histoire de l'art à réfléchir sur l'apparition d'œuvres non conformes au goût classique et sur l'unité stylistique de ces œuvres aberrantes. L'art baroque n'est donc pas réductible à un dialecte du classique au sens dédaigneux où l'entend Burckhardt, ni à une autre langue au sens un peu confus où Wölfflin l'emploie. Il relève d'un usage mineur, d'une minoration active de l'art reçu comme majeur dans la culture 
du temps. Cela nous renseigne sur la vie des normes dans la culture, en même temps que cela nous donne un principe méthodologique en histoire de l'art. La définition que Deleuze donne du style explique pourquoi l'histoire de l'art signale d'abord les mutations du goût par un jugement valoriel dépréciatif. La nouveauté du style surgit dans une culture historique, comme une contestation de la norme en vigueur, et ce faisant, elle souligne sa fécondité adversative. Norme classique doit s'entendre ici comme norme quelconque, il suffit qu'elle se pose comme majeure, qu'elle s'impose pragmatiquement. Cela ne définit ni une essence de l'art, ni même un statut déterminé de l'art dans la culture, pas plus qu'un répertoire morphologique, seulement une valeur normative, qui peut être très variable, mais qui produit sa minoration par son efficace même. Le statut du baroque s'explique par sa situation contemporaine de la naissance de l'historiographie. L'intérêt pour l'art s'y montre solidaire d'un goût du passé, d'un goût modelé et fortifié par la connaissance, la conservation et la jouissance du passé, et c'est dans cette tradition que la notion d'un art «baroque» pouvait prendre consistance. Cela indique la solidarité étroite entre l'épanouissement de la norme classique et l'historiographie d'art. S'en dégage un principe sur les conditions de l'histoire de l'art: elle est produite positivement par une norme active, solidaire d'un passé (d'une définition de la civilisation qui se conserve comme tradition), excluant de son champ ce qui ne relève pas de sa norme. L'histoire de l'art, au double sens descriptif et normatif que nous avons dégagé, résulte bien d'une productivité de la norme, au sens où Michel Foucault la définit, lorsqu'il applique les travaux de Canguilhem sur la norme vitale à la théorie de la culture. La norme classique n'est pas négative, elle est productrice. Il y a une productivité de la norme ${ }^{60}$ dans la mesure même où cette rétrospection valide, sur un "mode défensif ${ }^{61}$ ", une norme du goût et de la création (comprenant des recettes et des façons de produire, autant que des justifications intellectuelles, et des enjeux de pouvoir). De ce point de vue, l'art actuel participe tout autant de ce même champ de force. Mais à cause de cela même, une théorie du baroque ne pouvait pas voir le jour ${ }^{62}$. Cela ne signifie ni que le baroque soit anormal en lui-même, ni qu'il échappe à l'écriture: simplement, son anomalie le rendait invisible. Ceux des contemporains qui rédigeaient des traités s'identifiaient aux classiques, et n'entendaient aucunement valoriser une norme nouvelle. Les théoriciens qui entendaient proscrire certains artistes comme hors norme les passaient sous silence ou les considéraient comme insignifiants.

À la différence de la Renaissance, le baroque ne s'accompagne d'aucune théorie. Le style se développe sans modèles. On ne semble pas avoir eu le sentiment d'emprunter de nouvelles voies par principe. C'est pour cela qu'on ne donne pas alors de nom précis à ce style ${ }^{63}$.

Il faut bien le recul de l'histoire, joint à la manie de la conservation pour que ces œuvres réelles fassent craquer le cadre théorique de la théorie classique. L'art «baroque " naît bien d'une vue rétrospective, qui résulte du croisement entre cette historiographie normative, qui enjoignait à Quatremère de l'exclure, et le résultat positif de cette norme active, entraînant un goût de la collection, un statut pour les œuvres, couplant permanence et historicité. C'est pourquoi le baroque «est un long moment de crise ${ }^{64}$ » qui devait faire apparaître, $d u \mathrm{XV}^{\mathrm{e}}$ au $\mathrm{XIX}^{\mathrm{e}}$ siècle, qu'on ne peut réduire la production culturelle à la répétition du même. De ce point de vue, il faut donner raison à Quatremère de Quincy: le baroque est bien produit par la multiplication des chefs-d'œuvre, mais moins par l'épuisement d'une civilisation que par l'engorgement de la conscience historique. 

dégénérescence, mais une transformation active qui montre la puissance de la vie à l'œuvre dans la culture. Par cette vertu de transformation, toute œuvre significative, entendue par Deleuze comme œuvre mineure, déçoit les attentes du goût historique. Deleuze peut penser la création en art comme l'usage mineur d'une norme quelconque, parce qu'il passe d'une épistémologie de la norme comme essence invariante à une variabilité, une fluctuation immanente des normes au sein de la culture, tout en se situant dans la perspective d'une culture historique, qui collecte les échantillons du passé et statue sur le passage du temps.

31 Cela explique l'actualité baroque pour la philosophie actuelle, qui réfléchit sur le devenir, la mutation des cultures et la variation des goûts. La question du baroque montre l'historicité de notre rapport à l'art. C'est pourquoi Deleuze en fait la clé d'une philosophie de l'histoire, de l'art, et de la variation. Si Deleuze consacre en 1988 un livre à la question du baroque, comme concept philosophique, valant pour l'histoire de la philosophie (la philosophie de Leibniz) autant que pour la philosophie de l'histoire (réfléchissant sur le rapport entre les sciences, les arts et les philosophies), il s'agit autant de déterminer ce que l'âge baroque apporte à la philosophie actuelle que ce que l'art baroque apporte à l'art en général ${ }^{65}$. Pour autant, il ne s'agit pas d'isoler une essence du baroque, mais son concept, dans la perspective sous laquelle sa spécificité opère avec nous. En s'appuyant sur les travaux de Wölfflin, Deleuze montre que le baroque est « l'art de l'informel par excellence » parce qu'il « comprend les textures de la matière ${ }^{66}$ \%. Car le baroque s'intéresse aux manières par lesquelles la matière fait varier la forme. Contrairement à l'art des structures qui règle l'art renaissant, l'art baroque joue sur les textures, et remplace l'opposition de la matière et de la forme par la modulation des matériaux et des forces. Ainsi, «le couple fond-manière détrône la forme ou l'essence ${ }^{67} »$.

Tout contour s'estompe au profit des puissances formelles du matériau, qui montent à la surface et se présentent comme autant de détours et de replis supplémentaires ${ }^{68}$.

Deleuze entend justement substituer le concept de manière (d'être) à celui d'essence, c'est à dire passer d'une théorie de la norme majeure à une philosophie des variations. Le maniérisme, mis en œuvre dans Le Pli, doit être mis en corrélation avec l'art mineur ${ }^{69}$. Ainsi, le baroque ne recouvre pas les problèmes des mutations de la production d'art, du statut méthodologique de l'histoire de l'art, sans exiger une théorie épistémologique de la norme, qui fonde philosophiquement le statut d'une variation qui a d'abord été réfléchie comme une tératologie. Il y va d'une nouvelle réflexion sur la norme, qui s'inscrit, chez Deleuze, dans le passage du concept de " minorité » à celui de "maniérisme», entendu non tant comme description d'un style $^{70}$ que, sur le plan de la création en art, comme le passage d'une norme majoritaire dominante à sa minoration, son affaiblissement créateur. Cette théorie de la création est solidaire, sur le plan philosophique, du passage du concept d'essence à celui de manière, que Deleuze observe chez Leibniz. C'est ainsi que le baroque pose éminemment le problème de la variation. 


\section{BIBLIOGRAPHIE}

Alpers Svetlana, « Roger de Piles », in Édouard Pommier (éd.), Histoire de l'histoire de l'art, t. 1, « De l'Antiquité au XVIII ${ }^{\mathrm{e}}$ siècle », cycle de conférences organisé au musée du Louvre, Paris, Louvre, Klincksieck, 1995.

Argan Giulio Carlo, L’Âge baroque [1964], réédition Genève, Skira, 1989.

Argan Giulio Carlo, L'histoire de l'art et la ville. Crise, culture, design [1983], Claire Fargeot (trad.), Paris, Éditions de la Passion, 1995.

Belting, Hans, L'histoire de l'art est-elle finie ? [1983], Jean-François Poirier et Yves Michaud (trad.), Nîmes, Jacqueline Chambon, 1989.

Bourdieu Pierre, Les règles de l'art. Genèse et structure du champ littéraire, Paris, Seuil, 1992.

Burckhardt Jacob, Der Cicerone. Eine Einleitung zum Genuss der Kunstwerk Italiens, Stuttgart, réédition Gesammelte Werke, Bd IX, Bâle, Benno Schwabe \& Co Verlag, 1860.

Deleuze Gilles, « Philosophie et Minorité », in Critique, n 34, février 1978.

Deleuze Gilles, Foucault, Paris, Minuit, 1986.

Deleuze Gilles, Le Pli. Leibniz et le Baroque, Paris, Minuit, 1988.

Deleuze Gilles, Guattari Félix, Kafka. Pour une littérature mineure, Paris, Minuit, 1975.

Deleuze Gilles, Guattari Félix, Capitalisme et schizophrénie, t. 2, Mille plateaux, Paris, Minuit, 1980.

Dictionnaire de l'Académie française, $1^{\mathrm{re}}$ édition $1694,2^{\mathrm{e}}$ édition 1740.

Dvořák Max, "Über Greco und den Manierismus”, in Kunstgeschichte als Geistesgeschichte, Munich, 1928.

Foucault Michel, «Qu'est-ce qu'un auteur? », Bulletin de la société française de philosophie, in Dits et Écrits, $63^{\mathrm{e}}$ année, $\mathrm{n}^{\circ} 3$, juillet-septembre 1969.

Furetière Antoine, Dictionnaire universel, contenant généralement tous les mots françois tant vieux que modernes et les termes de toutes les sciences et des arts [1684], [1690], réédition en fac-similé, 1978.

Gombrich Ernst Hans, "Norm and Form. The Stylistic Categories of Art History and their Origins in Renaissance Ideals", in Norm and Form: Studies in the Art of the Renaissance, Londres, Phaidon, 1968.

Hildebrand Adolf (von), Das Problem der Form in der bildenden Kunst, Strasbourg, 1893, traduction française Éliane Beaufils, Paris, L'Harmattan, 2002.

Hume David, Les essais esthétiques, traduction et introduction par Renée Bouveresse, Paris, Vrin, 1973.

Imbs Paul (éd.), Trésor de la langue française. Dictionnaire de la langue du XIX et du XXe siècle, Paris, Éd. du CNRS, 1975.

Kant Emmanuel, Critique de la faculté de juger, 1790.

Lomazzo Giovanni Paolo, Trattato dell'Arte, Milan, 1585.

Macherey Pierre, «Pour une histoire naturelle des normes », in Michel Foucault philosophe.

Rencontre internationale, Paris, 9, 10, 11 janvier 1988, Paris, Seuil, 1989. 
Macherey Pierre, «De Canguilhem à Canguilhem en passant par Foucault », in Georges Canguilhem. Philosophe, historien des sciences, actes du colloque des 6, 7, 8 décembre 1990, Paris, Albin Michel, 1993.

Milizia Francesco, Dictionnaire des beaux-arts, Milan, Pietro Agnelli, $1^{\text {re }}$ édition $1797,2^{\mathrm{e}}$ édition 1804.

Migliorini Bruno, Manierismo, barocco, rococo, Rome, Academia Nazionale dei Lincei, 1962.

Nietzsche Friedrich, De l'utilité et des inconvénients de l'histoire pour la vie, " deuxième considération inactuelle », 1874.

Panofsky Erwin, «Le concept du Kunstwollen » [1920], in La perspective comme forme symbolique et autres essais, traduction française sous la direction de Guy Ballangé, Paris, Minuit, 1975.

Panofsky Erwin, Idea. Contribution à l'histoire du concept de l'ancienne théorie de l'art [1924], traduction française Henri Joly, Paris, Gallimard, 1983, réédition 1989.

Podro Michael, Les historiens d'art [1982], traduction française (pas de nom de traducteur mentionné), Saint-Pierre-de-Salerne, Gérard Montfort, 1990.

Quincy Quatremère (de), Dictionnaire historique d'architecture. Comprenant dans son plan les notions historiques, descriptives, archéologiques, biographiques, théoriques, didactiques et pratiques de cet art, Paris, librairie d'Adrien le Clere et $C^{\text {ie }}$, quai des Augustins, 1832.

Rousset Jean, La littérature de l'âge baroque en France. Circé et le paon, Paris, José Corti, 1953.

Schlosser Julius (von), La littérature artistique. Manuel des sources de l'histoire de l'art moderne, Vienne [1924], Jacques Chavy (trad.), Paris, Flammarion, 1984.

Simondon Gilbert, L'individu et sa genèse physico-biologique. L'individuation à la lumière des notions de forme et d'information, Paris, PUF (Épiméthée), 1964, réédition complétée, Grenoble, Jérôme Millon (Krisis), 1995.

Simone Franco, « Première histoire de la périodisation du baroque », in Renaissance Maniérisme Baroque, actes du XI ${ }^{\mathrm{e}}$ stage international de Tours, Paris, Vrin, 1972.

Tapié Victor-Lucien, Baroque et classicisme [1957], Paris, Le Livre de poche (Pluriel), 1980. Vasari Giorgio, Le Vite de'più eccelenti pittori, scultori ed architettori, Les vies des meilleurs peintres, sculpteurs et architectes, traduction française et publication critique sous la direction d'André Chastel, 12 vol., Paris, Berger-Levrault, 1981, 1983, 1989.

Winckelmann Johann Joachim, Geschichte der Kunst des Altherthums (Histoire de l'art dans l'Antiquité) [1934], Ludwig Goldscheider (ed.), Vienne, Phaidon Verlag, traduction française (édition d'Amsterdam, 1766, fac-sim.), Genève, Minkoff reprint, 1972.

Wölfflin Heinrich, Renaissance und Barock: eine Untersuchung über Wesen und Entstehung des Barockstils in Italien, Leipzig, Koehler \& Amelang, 1986.

Wölfflin Heinrich, Renaissance et baroque [1888], traduction française Guy Ballangé, Paris, Livre de Poche, 1985, réédition Gérard Montfort, 1988.

Wölfflin Heinrich, Réflexions sur l'histoire de l'art [1941], Rainer Rochlitz (trad.), Flammarion, 1997. Zerner Henri, Écrire l'histoire de l'art, Jeanne Bouniort (trad.), Paris, Gallimard (Art et artistes), 1997. 


\section{NOTES}

1. Cf. Pierre Bourdieu, Les règles de l'art. Genèse et structure du champ littéraire, Paris, Seuil, 1992, p. 401. Reste à déterminer comment une telle analyse historique peut être menée à bien. Deleuze montre qu'une "nouvelle philosophie de l'histoire" (il pense au travail de Foucault) doit être comprise comme réduction de la phénoménologie à l'épistémologie : le phénomène, y compris le phénomène sociologique, doit faire l'objet d'une constitution qui est de l'ordre du savoir: Gilles Deleuze, Foucault, Paris, Minuit, 1986, p.117. Car « le principe le plus général de Foucault est: toute forme est un composé de rapport de forces " (ibid., p. 131) et ceci est valable pour le plan sociologique comme pour le plan intellectuel. Bourdieu a donc raison de déclarer que « rien n'est moins naturel que l'attitude à adopter devant une œuvre d'art » et d'insister sur la constitution sociohistorique du champ esthétique, mais non de réifier la strate sociologique en considérant les rapports de pouvoir à leur tour comme des essences.

2. Cette thématique de l'individuation, appliquée par Deleuze aux objets de la culture, lui vient des travaux de Simondon (cf. Gilbert Simondon, L'individu et sa genèse physico-biologique. L'individuation à la lumière des notions de forme et d'information, Paris, PUF, (Épiméthée), 1964, réédition complétée, L'individu et sa genèse physico-biologique, Grenoble, J. Millon (Krisis), 1995). Foucault s'en montre proche lorsqu'il parle de «l'individualisation dans l'histoire des idées » et qu'il regrette que l'histoire de la culture, qu'il s'agisse de l'histoire littéraire ou philosophique, soit portée par des notions non élucidées comme celle de l'auteur et de l'œuvre, alors que toute histoire critique - toute philosophie - devrait d'abord commencer par chercher «comment l'auteur s'est individualisé dans une culture comme la nôtre " (cf. «Qu'est-ce qu'un auteur?», Bulletin de la société française de philosophie, $63^{\mathrm{e}}$ année, $\mathrm{n}^{\circ}$ 3, juillet-septembre 1969, p. 73-104, in Dits et Écrits, I, p. 789-821; la citation se trouve p. 792).

3. C'est deux ans après la parution du livre consacré à Foucault que Deleuze livre sa théorie du baroque : Gilles Deleuze, Le Pli. Leibniz et le Baroque, Paris, Minuit, 1988. Dans l'œuvre de Foucault, Deleuze s'intéresse aux conditions d'une philosophie de l'histoire - déterminer «les combinaisons variables des deux formes, le visible et l'énonçable » (G. Deleuze, Foucault, op. cit., p. 90) qui constituent le savoir, dans son historicité. Deleuze en attend les conditions philosophiques d'une rupture avec la phénoménologie. Il souligne l'importance de Heidegger pour Foucault, en insistant néanmoins sur leur différence : «le pli et le dépli ont chez Foucault une origine, un usage, une destination très différents de ceux de Heidegger » (Ibid., p. 137). Réfléchir sur l'unité conceptuelle d'une strate historique (l'âge baroque), en cherchant les conditions d'une «ligne» qui permette de penser ensemble arts, sciences et philosophie, une ligne commune aux «architectes, peintres, musiciens, poètes, philosophes » (Le Pli, p. 48); faire $\mathrm{du}$ « concept opératoire du baroque » le « pli » indique suffisamment la continuité entre les deux ouvrages. Déterminer sous quelles conditions un art baroque devient «visible » s'inscrit donc dans la perspective foucaldienne des travaux de Deleuze. (Le visible, chez Foucault, ne se limite pas à la vue, mais couvre le perceptible en général, comme l'indique Deleuze : « Les visibilités ne se définissent pas par la vue, mais sont des complexes d'actions et de passions, d'actions et de réactions, des complexes multi-sensoriels, qui viennent à la lumière», G. Deleuze, Foucault, p. 66).

4. Erwin Panofsky, «Le concept du Kunstwollen » [1920], in La perspective comme forme symbolique et autres essais, traduction sous la direction de Guy Ballangé, Paris, Minuit, 1975, p. 197-221: «Pour la critique d'art, c'est en même temps une bénédiction et une malédiction que ses objets (de science) émettent nécessairement la prétention d'être compris autrement que sous le seul angle historique ", p. 197. Il n'est pas sûr que l'effet esthétique puisse être ainsi opposé à la visibilité historiographique. Argan est mieux inspiré en notant, «l'histoire de l'art est la seule de toutes les histoires spécifiques qui se fasse en présence des événements et ne doive ni les évoquer, ni les reconstruire, ni les narrer mais seulement les interpréter ", cf. C. G. Argan, L'histoire de l'art et la ville. Crise, culture, design, 1983, Claire Fargeot (trad.), Paris, Éditions de la Passion, 1995, p. 16. 
Cette « interprétation » montre à quel point l'historiographie est constitutive de son objet. L'histoire de l'art exhibe ici une difficulté qui concerne aussi bien l'histoire générale.

5. Une épistémologie de la danse ou de la musique - des œuvres événementielles - serait obligée de prendre en compte ce problème supplémentaire: la transformation qu'impose leur transcription dans un médium durable.

6. On peut en effet soutenir simultanément que les conditions de l'esthétique sont données historiquement tout en exigeant de la philosophie qu'elle pense l'art sans s'en tenir au seul déterminisme sociohistorique, à condition de produire une théorie critique de l'histoire et de penser l'art comme effet vital : c'est ce que tentent, à la suite de Nietzsche, Foucault et Deleuze.

7. Comme l'indique, par exemple, Bourdieu, « [...] faut-il dire que c'est le point de vue esthétique qui crée l'objet artistique ou bien que ce sont les propriétés spécifiques et intrinsèques de l'œuvre d'art qui suscitent l'expérience esthétique [...] », Les règles de l'art, op. cit., p. 395. Bourdieu a raison de montrer qu'une telle alternative est trop large. On peut néanmoins considérer qu'il se range finalement à la première option en pensant l'art comme évolution du champ social objectif, structure de forces dont il revient à la sociologie scientifique de déterminer les invariants. On n'aurait aucun mal à situer la plupart des théoriciens s'intéressant au baroque dans la seconde voie : celle d'une essence anhistorique du baroque, soit comme dégénérescence (théorie sénile) soit comme rupture tonique avec la norme. Deleuze lui-même, quoiqu'il substitue à cette alternative une théorie de la variation, du maniérisme qui échappe à l'alternative, donne souvent l'impression de valoriser une essence du baroque. En fait, il s'agit d'un maniérisme qui remplace l'essentialisme - une nouvelle théorie de la perspective, jointe à une critique de l'histoire.

8. On appellera ici «classique » la norme pragmatique active qui unifie la production plastique depuis la Renaissance sous l'autorité de l'imitation des Antiques, et qui s'exprime par des prescriptions, modes de réception, apprentissage, recettes d'exécution unifiant le goût des artistes comme le goût collectif qui les instruit et sur lequel ils agissent en retour.

9. Sur Bellori, consulter J. von Schlosser, La littérature artistique. Manuel des sources de l'histoire de l'art moderne (Vienne, 1924), traduction française Jacques Chavy, Paris, Flammarion, 1984 et E. Panofsky, Idea. Contribution à l'histoire du concept de l'ancienne théorie de l'art (Leipzig, Berlin, 1924), traduction française Henri Joly, Paris, Gallimard, 1983, réédition 1989.

10. Cf. H. Wölfflin, Renaissance et baroque, 1888, traduction française Guy Ballangé, Paris, Livre de Poche, 1985, réédition Gérard Montfort, 1988, avec une excellente présentation de B. Teyssèdre. C'est cette édition que nous utilisons, nous reportant quand il y a lieu à l'édition allemande (Renaissance und Barock: eine Untersuchung über Wesen und Entstehung des Barockstils in Italien, Leipzig, Koehler \& Amelang, 1986).

11. Vasari, artiste lui-même, que nous qualifierions aujourd'hui de "maniériste ", successeur de Michel-Ange et créateur de la première académie florentine, est l'auteur en 1568 de la première monumentale histoire des arts plastiques de l'Italie renaissante : G. Vasari, Le Vite de'più eccelenti pittori, scultori ed architettori, Les vies des meilleurs peintres, sculpteurs et architectes, traduction française et publication critique sous la direction d'André Chastel, 12 vol., Paris, Berger-Levrault, 1981, 1983, 1989.

Winckelmann est l'auteur de la première histoire de l'art grec. Sa Geschichte der Kunst des Altherthums (Histoire de l'art dans l'Antiquité) paraît à Dresde en 1764. Les éditions utilisées sont J. J. Winckelmann, Geschichte der Kunst des Altertums, L. Goldscheider (éd.), Vienne, Phaidon Verlag, 1934 et, pour la traduction française, l'édition d'Amsterdam, 1766, fac sim., Genève, Minkoff reprint, 1972.

12. H. Wölfflin, Renaissance und Barock, p. 16.

13. «On peut dire avec certitude que l'art est allé aussi loin dans l'imitation de la nature qu'il est possible d'aller ; il s'est élevé si haut qu'il est à craindre de le voir s'abaisser plutôt qu'à espérer désormais le voir s'élever encore ", G. Vasari, Les vies, op. cit., t. 3, p. 19. 
14. Ou on les décrit seulement comme contrevenant au classique, en se réclamant des bons auteurs antiques (Vitruve en particulier) ; voir E. H. Gombrich, "Norm and Form. The Stylistic Categories of Art History and their Origins in Renaissance Ideals", in Norm and Form: Studies in the Art of the Renaissance, Londres, Phaidon, 1968, p. 83-89.

15. Wölfflin, comme tous les commentateurs, relève les emplois de bizzarro, stravagante, de capricciosi ornamenti que Vasari emploie pour faire la louange de Michel-Ange (H.Wölfflin, Renaissance und Barock, p. 41). Cela ne fait pas de Vasari le père fondateur du baroque pour autant. On trouve les mêmes qualificatifs chez Lomazzo, Trattato dell'Arte (Milan, 1585), considéré comme un théoricien du maniérisme.

16. G. Vasari, Les Vies, op. cit., Vie de Michel-Ange, cité par Wölfflin, Renaissance et baroque, p. 43.

17. Panofsky présente Bellori comme le "précurseur » de Winckelmann, en tant que spécialiste de l'Antiquité autant que comme théoricien du beau (notamment de la théorie du beau idéal in Geschichte der Kunst des Altertums, op. cit., IV, 2, § 33 sq.). C'est Bellori qui confère à la notion d'Idea « la forme sous laquelle elle s'introduira par la suite en France et en Allemagne », E. Panofsky, Idea, op. cit., p. 133-135.

18. En prenant appui sur un historien de l'art bien postérieur (Aloïs Riegl), Benjamin fait la théorie de ce rapport entre art baroque et histoire, mais surtout pour la littérature - le drame baroque (cf. W. Benjamin, L'origine du drame baroque allemand (composé en 1925, édité en 1974), traduction française Sibylle Muller, Paris, Flammarion, 1985, spécialement au chapitre « Allégorie et Trauerspiel »). Riegl étant le théoricien de l'art mineur, son importance pour le débat qui nous occupe ici est profonde. Benjamin montre (en prenant appui sur Riegl) que la pauvreté des œuvres du drame baroque allemand n'empêche pas l'intérêt très vif de l'étude du genre baroque. Il y a une vie des normes, indépendante de l'histoire des chefs-d'œuvre. Si le baroque se signale pour une telle étude, ce n'est pas négativement, à cause de l'indigence de ses productions, mais parce que cette indigence révèle exemplairement, comme le fait le passage du symbole à l'allégorie, le passage d'une nature de l'art à une histoire de l'art. Comme Deleuze l'explique très bien, «Walter Benjamin fit faire à la compréhension du Baroque un progrès décisif lorsqu'il montra que l'allégorie n'était pas un symbole raté, une personnification abstraite, mais une puissance de figuration tout à fait différente de celle du symbole : celui-ci combine l'éternel et l'instant, presque au centre du monde, mais l'allégorie découvre la nature et l'histoire suivant l'ordre du temps, elle fait de la nature une histoire et transforme l'histoire en nature, dans un monde qui n'a plus de centre », G. Deleuze, Le pli, op. cit., p. 170-171.

19. V.-L. Tapié, Baroque et classicisme, Paris, 1957, réédition Le Livre de poche (coll. «Pluriel »), 1980, p. 49; voir aussi F. Simone, "Première histoire de la périodisation du baroque », in Renaissance Maniérisme Baroque, actes $\mathrm{du} \mathrm{xI}^{\mathrm{e}}$ stage international de Tours, Paris, Vrin, 1972, p. 28-38, ainsi que la mise au point ancienne mais pertinente de Jean Rousset, La littérature de l'âge baroque en France. Circé et le paon, Paris, José Corti, 1953, p. 281-286, pour son historique du baroque plastique et son application des principes de Wölfflin à la littérature. Pour des références plus actualisées, consulter l'excellente « présentation » que Bernard Teyssèdre donne à l'édition française de Wölfflin, Renaissance et Baroque, op. cit.

20. Attesté depuis la fin du XIII ${ }^{\mathrm{e}}$ siècle sous la forme barroca, cf. Paul Imbs (éd.), Trésor de la langue française. Dictionnaire de la langue du XIX et du XX siècle, Paris, Éd. du CNRS, 1975, t. 4, p. 201.

21. «C'est un terme de joaillerie qui ne se dit que de perles qui ne sont pas parfaitement rondes ", Antoine Furetière, Dictionnaire universel, contenant généralement tous les mots françois tant vieux que modernes et les termes de toutes les sciences et des arts, 1684, 1690, réédition en fac sim., 1978.

22. «Baroque, adjectif. Se dit seulement des perles qui sont d'une rondeur fort imparfaite. Un collier de perles baroques ", Dictionnaire de l'Académie française, $1^{\text {re }}$ édition de 1694, cité par Tapié, p. 56. 
23. Attesté selon le Trésor de la langue française, op. cit., depuis 1718 au sens de «bizarre, choquant ». Une perle qui ne correspond pas aux normes de la joaillerie.

24. Dictionnaire de l'Académie française, édition de 1740, cité par Tapié, p.56. La collision sémantique entre le «baroque » et le «bizarre » est très intéressante, le bizarre (de l'espagnol bigearre, étrange) ayant d'abord l'acception "d'audace merveilleuse» avant de recevoir celle de «singularité grotesque» (Tapié, op. cit., p. 57). Cela confirme la compréhension kantienne du génie comme exception de la norme, conception qui s'applique parfaitement à la réception de Michel-Ange par ses contemporains et explique l'admiration éperdue que lui voue Vasari, lorsqu'il « salue comme une délivrance » son « audace inouïe » (H. Wölfflin, Renaissance et baroque, p. 43).

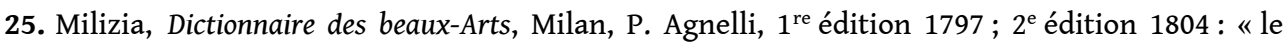
paroxysme du bizarre, le comble du ridicule », cité par Teyssèdre, "présentation », in wölfflin, Renaissance et baroque, p. 7, Tapié, op. cit., p. 57.

26. B. Migliorini, Manierismo, barocco, rococo, Rome, Academia Nazionale dei Lincei, 1962, p. 46-47.

27. Quatremère de Quincy, Dictionnaire historique d'architecture. Comprenant dans son plan les notions historiques, descriptives, archéologiques, biographiques, théoriques, didactiques et pratiques de cet art, Paris, librairie d'Adrien le Clere et $C^{\text {ie }}$, quai des Augustins, 1832. Sur la reprise de la même définition, cf. Tapié, p. 56-58.

28. De même que Benjamin montre que l'allégorie baroque diffère du symbole, Deleuze explique qu'il s'agit, avec l'âge baroque, lorsqu'on réfléchit sur ces mutations du sens dans l'histoire, de passer d'une théorie de la métaphore - qui implique un passage du propre à la figure - à une théorie de la métamorphose. La critique de la métaphore s'effectue dans G. Deleuze et F. Guattari, Kafka. Pour une littérature mineure, Paris, Minuit, 1975 : « Il n'y a plus désignation de quelque chose d'après un sens propre, ni assignation de métaphores d'après un sens figuré. Mais la chose comme les images ne forment plus qu'une séquence d'états intensifs [...]», p. 39. Sans entrer ici dans la théorie complexe de l'intensif chez Deleuze, observons, pour le problème qui nous intéresse, la migration du sens d'un mot dans la langue, et son usage figuré, que s'y joue un même problème : celui de la fluctuation autour de la norme.

29. E. Kant, Critique de la faculté de juger, § 45-49 (AK, V, 306-319). À comparer avec le pessimisme épuisé de Hildebrand: "C'est une des caractéristiques de notre âge scientifique qu'une œuvre d'art positive ne dépasse pas le stade de l'imitation ", A. von Hildebrand, Das Problem der Form in der bildenden Kunst, Strasbourg, 1893. p. IX, cité par M. Podro, Les historiens d'art (1982), traduction française, sans nom de traducteur, Saint-Pierre-de-Salerne, Gérard Montfort, 1990, p. 58.

30. G. Deleuze, Le Pli, p. 47.

31. Quatremère de Quincy, Dictionnaire historique d'architecture, op. cit., p. 159.

32. Ibid., art. BAROQUE, p. 159.

33. Ibid., art. BIZARRERIE, p. 205.

34. Ibid., p. 205.

35. «Il est vrai que ces modèles sont invariables; mais ce qui peut toujours varier, c'est le goût de ceux qui les reproduisent, et la fantaisie de ceux qui en jugent l'imitation », Ibid., p. 205-206.

36. Le "style archaïque", expressif mais raide, des premiers âges débouche sur le «style grandiose ou sublime » de Phidias et de Polyclète, l'âge d'or de l'Athènes de Périclès, mais avec le «beau style » de Lysippe et Apelle, le « haut style » perd de sa noblesse, devient plus gracieux et plaisant, et débouche finalement sur le "style d'imitation ", qui marque l'affadissement de la règle et sa dissolution par l'éclectisme et la virtuosité, qui indiquent la perte de la norme (J. J. Winckelmann, Histoire de l'art dans l'Antiquité, op. cit., tome II, partie I, chapitre $4,3^{\mathrm{e}}$ section, p. 2-3; les qualificatifs employés sont le «ältere Stil », le « grosse und hohe Stil »; le "schöne Stil », le "Stil der Nachahmer», cf. Geschichte der Kunst des Altertums, op. cit., p. 207). C'est dans le chapitre consacré à la corruption du goût hellénistique, à son excès pédant de raffinement, que Winckelmann exécute les artistes baroques comme Borromini et le Bernin : « ils s'éloignent de la 
nature et de l'Antiquité, dans la peinture, la sculpture et l'architecture ». Voir J. J. Winckelmann, Histoire de l'art dans l'Antiquité, tome II, p. 233 ; Geschichte der Kunst des Altertums, p. 335.

37. Le style d'imitation traduit la perte de vitalité artistique par la dégénérescence des formes : la copie, l'effusion décorative, la ligne ornementale remplacent l'étude de la belle nature, mais cette dégénérescence du style, à l'œuvre dès le troisième moment, témoigne de la corruption politique et morale de la civilisation hellène, vaincue par Alexandre, J. J. Winckelmann, Histoire de l'art dans l'Antiquité, op. cit., tome II, p. 229 : «On entend par âge de la décadence de l'art celui des artistes qui se distinguèrent après la mort d'Alexandre ", Geschichte der Kunst des Altertums, p. 333.

38. Quatremère de Quincy, Dictionnaire historique d'architecture, op. cit., art. BORROMINI, p. 217.

39. On appelle « confession » l'« autel placé sur les corps des martyrs », explique Quatremère.

40. Quatremère de Quincy, Dictionnaire historique d'architecture, op. cit., art. BERNIN, p. 194.

41. Ibid., p. 204.

42. Ibid., p. 206.

43. Quatremère reprend une théorie de la dégénérescence qui lie la décadence des arts non à la corruption des mœurs mais à une corruption du goût par excès de civilisation. Les arts dégénèrent par excès de raffinement. Cf. $\mathrm{D}$. Hume, De la simplicité et du raffinement dans l'art d'écrire, 1742 : «C'est l'extrême dans lequel les hommes sont les plus aptes à tomber une fois que la culture a fait quelque progrès et que des écrivains éminents sont apparus dans tous les genres littéraires [...] la tentative pour plaire par la nouveauté éloigne les hommes de la simplicité de la nature, et remplit leurs écrits d'affectation et de pointes ", in D. Hume, Les essais esthétiques, traduction et introduction par Renée Bouveresse, Paris, Vrin, 1973, t. 2, p. 78. On trouverait des déclarations analogues chez Rousseau. On a vu que Winckelmann appliquait ces théories à l'histoire de l'art de la Grèce.

44. Quatremère de Quincy, Dictionnaire historique d'architecture, op. cit., art. BIZARRERIE, p. 204. Il s'agit des premières lignes de l'article.

45. Cf. E. Kant, Critique de la faculté de juger, op. cit., § 49, AK, V, p. 318 : Le génie est défini chez Kant comme cette subjectivité capable de faire résonner directement la voix de la nature sans passer par la médiation de la culture, puisque le génie est cette subjectivité capable de se soustraire à l'influence de la culture (à l'imitation) pour se faire l'écho de « la règle de la nature ». Par sa définition du sujet créateur, Kant lie pour longtemps la valeur en art (théorie du chefd'œuvre) à cette vertu provocante, par laquelle les règles du genre, les normes du goût sont transgressées. Kant télescope ces deux moments du goût, subjectif naturel et collectif social, parce qu'il pense le « Génie » comme « le talent par lequel la Nature donne sa règle à l'art ». Pour Kant, implicitement, cette norme de la nature est la norme de la beauté elle-même. Mais Kant dit bien, puisqu'il refuse que l'art se réduise à l'imitation, seulement technique, des œuvres du passé, et puisqu'il distingue entre l'imitation qui est la règle de l'apprentissage, affaire d'écolier, affaire d'apprenti et la véritable création, qu'il doit y avoir une constitution des nouvelles règles dans la culture : «On voit par là que le génie : 1) est un talent, qui consiste à produire ce pour quoi on ne saurait donner aucune règle déterminée"; sa première caractéristique (Eigenschaft) est l'originalité (Originalität), mais une originalité qui puisse être érigée en modèle (Model) sans verser dans l'absurde (Unsinn), d'où le concept d'une originalité « exemplaire » (exemplarisch).

46. Cf. F. Nietzsche, De l'utilité et des inconvénients de l'histoire pour la vie, deuxième considération inactuelle, 1874.

47. H. Wölfflin, Renaissance et baroque, op. cit., p. 33.

48. Ibid., p. 35.

49. H. Wölfflin, Renaissance und Barock, p. 14 : Die Formanalyse "muss zeigen, welcher Komplex von Symptomen den Barock konstituiert". C'est une symptomatologie du passage d'une norme à l'autre : cf. introduction. L'art antique " mourant » présente des symptômes analogues ("ähnliche Symptomen", Ibid., p. 11 et "Diese Symptomen aufzusuchen, ist unsere Aufgabe" (« Rechercher ces symptômes, telle est notre tâche ») (Ibid., p. 12). 
50. H. Wölfflin, Renaissance et baroque, p. 31, traduction modifiée. Le texte allemand est le suivant : "Meine Absicht war, die Symptome des Verfalls zu beobachten und in der 'Verwilderung und Willkür' womöglich das Gesetz zu erkennen, das einen Einblick in das innere Leben der Kunst gewähre", Renaissance und Barock, p. 10.

51. Wölfflin dit lui-même que c'est à son maître, Jakob Burckhardt, qu'il doit l'intérêt qui l'a porté vers le baroque (Renaissance et baroque, $2^{\mathrm{e}}$ édition, 1906).

52. J. Burckhardt, Der Cicerone. Eine Einleitung zum Genuss der Kunstwerk Italiens, Stuttgart, réédition Gesammelte Werke, Bd IX, Bâle, Benno Schwabe \& Co Verlag, 1860, p. 305 : "Die Barockbaukunst spricht dieselbe Sprache wie die Renaissance, aber einen verwilderten Dialeckt davon".

53. H. Wölfflin, Réflexions sur l'histoire de l'art (1941), R. Rochlitz (trad.), Flammarion, 1997, p. 35.

54. Le substantif est formé d'après le verbe verwildern, qui se dit des animaux domestiques qui retournent à l'état sauvage, d'un jardin ou d'un enfant qu'on laisse à l'abandon.

55. En fait, Wölfflin oscille perpétuellement entre deux positions : le baroque est une langue formelle propre, mais il constitue aussi « un progrès de la vision classique ». Il juxtapose le thème de la dégénérescence sénile à celui de la langue formelle propre, plus qu'il ne les sépare. C'est qu'il reste pris dans la perspective d'une succession des âges classique et baroque au sein de la seule tradition occidentale, et qu'il n'est pas loin de considérer ces deux styles comme épuisant le destin de l'art en général. Cela n'ôte rien à la vigueur de son analyse du baroque.

56. C'est pourquoi son entreprise elle-même a pu être rapprochée de la tentative de Saussure, cf. S. Alpers, « Roger de Piles ", in Édouard Pommier (éd), Histoire de l'histoire de l'art, t. 1, De l'Antiquité au XVIII ${ }^{\mathrm{e}}$ siècle : cycle de conférences organisé au musée du Louvre, Paris, Louvre, Klincksieck, 1995, p. 285-301.

57. Les textes principaux se trouvent dans G. Deleuze et F. Guattari, Pour une littérature mineure, op. cit., G. Deleuze, "Philosophie et Minorité", in Critique, n 34, février 1978, p. 154-155, G. Deleuze et F. Guattari, Capitalisme et schizophrénie, t. 2, Mille plateaux, Paris, Minuit, 1980.

58. E. H. Gombrich, "Norm and Form”, op. cit., p. 81-86, et spécialement p. 82.

59. Dans la première édition des Vies de Bellori, la Vie du Caravage est précédée d'un frontispice représentant l'image d'une vieille femme, allégorie (comme l'explique la légende) de la praxis, que la théorie maniériste opposait à la théorie, comme le moment pratique (le métier, dévalorisé) opposé au moment intellectuel de l'art. Cela signifie que pour le Caravage, la pratique de l'exécution seule compte, et non la théorie, modèle historique des Anciens, invention ou imagination. Cf. G. C. Argan, L’Âge baroque, 1964, réédition Genève, Skira, 1989, p. 121.

60. P. Macherey, «Pour une histoire naturelle des normes», in Michel Foucault philosophe. Rencontre internationale, Paris, 9, 10, 11 janvier 1988, Paris, Seuil, 1989, p. 203-221, p. 210 ; également, "De Canguilhem à Canguilhem en passant par Foucault", in Georges Canguilhem. Philosophe, historien des sciences, actes du colloque des 6, 7, 8 décembre 1990, Paris, Albin Michel, 1993, p. 286-294.

61. H. Belting, L'histoire de l'art est-elle finie? [1983], traduction française J.-F. Poirier et Y. Michaud, Nîmes, Jacqueline Chambon, 1989, p. 102.

62. H. Belting, op. cit., p. 104-106 l'explique très clairement : « Les normes jumelles du classicisme des Grecs et des Florentins décourageaient tout travail ultérieur. Une théorie historique de l'art baroque n'a jamais pu voir le jour, elle ne relevait certainement pas de la perspective de Vasari. La littérature sur l'art inclina nettement vers un classicisme très partial et elle sélectionna strictement les productions contemporaines, quand elle ne leur devenait pas, consciemment ou inconsciemment, étrangère. L'art devint ainsi une chaîne de nouvelles naissances niant indéfiniment le déclin du classique [...] Les Vies de Vasari livraient plus des normes artistiques qu'un modèle pour écrire l'histoire. Ces normes servaient encore chez Winckelmann à polémiquer contre l'art de son temps ».

63. H. Wölfflin, Renaissance et baroque, op. cit., p. 41.

64. G. Deleuze, Le Pli, p. 91.

65. Ibid., p. 48. 
66. Ibid., p. 49.

67. Ibid., p. 72.

68. Ibid., p. 23.

69. Ibid., p. 166.

70. Le maniérisme est un dérivé de la «manière » qui, chez Vasari, est synonyme de style. La «manière » est supplantée par le vocable plus neutre de "style» au XVII ${ }^{\mathrm{e}}$, sous l'influence de Bellori, parce qu'elle renvoie trop à la manière singulière, et semble porter un risque d'affectation, d'arbitraire individuel, de trop grande variation.

En histoire de l'art, le maniérisme désigne la manière (le style) qui, chez certains peintres de la génération succédant à Raphaël (Rosso, Pontormo, Parmesan), indique une mutation du cadre renaissant. Les cadrages, l'éclairage, le choix des teintes acidulées ne relèvent plus de l'idéalisation plastique de la nature. Vasari, Michel-Ange lui-même, appartiennent à cette période de la fin de la Renaissance, ou du début de l'âge baroque. C'est dans les années vingt que Max Dvořák isole cette période sous le nom de "maniérisme », lui accordant par cette nomination la consistance d'un style autonome et non d'une variante tardive; “Über Greco und den Manierismus", in Kunstgeschichte als Geistesgeschichte, Munich, 1928, p. 261-276, cité par Henri Zerner, Écrire l'histoire de l'art, traduction française Jeanne Bouniort, Paris, Gallimard (Art et artistes), 1997, p. 134. Voir E. Panofsky, Idea. Contribution à l'histoire du concept de l'ancienne théorie de l'art, op. cit., p. 126, et n ${ }^{\circ} 244$, p. 259-251.

\section{RÉSUMÉS}

Loin d'être une simple étiquette descriptive, la catégorie stylistique engage les facteurs du goût et du jugement de valeur, de la culture et de l'histoire. Exemplaire de ce point de vue est la constitution du «baroque ». Mais de Quatremère de Quincy à Wölfflin, de la tératologie du baroque à sa morphologie, l'émergence et les modifications de cette notion stylistique témoignent de la difficulté à penser l'écart par rapport à la norme (ici : l'antique, le classique, la Renaissance). Le baroque, comme catégorie, est exemplaire de ce travail des normes, dans leur aspect constructif autant que normatif: une épistémologie de l'histoire de l'art montre la manière dont varient les normes et les œuvres qui les mettent en variation.

\section{INDEX}

Mots-clés : baroque, écart, goût, norme, style, valeur, variation

Personnes citées : Quatremère de Quincy, Wölfflin (Heinrich), Deleuze (Gilles)

\section{AUTEUR}

\section{ANNE SAUVAGNARGUES}

Professeur de philosophie de l'art à l'université Paris X Nanterre, Anne Sauvagnargues dirige le département de philosophie et la sous-équipe Esthétique de l'EA HAR 4414.Spécialiste de philosophie contemporaine, en particulier de Deleuze, elle a notamment publié, « Deleuze. De 
l'animal à l'art », in Paola Marrati, Anne Sauvagnargues et François Zourabichvili, La philosophie de Deleuze, PUF, 2004 ; Deleuze et l'art, PUF, 2005 et Deleuze. L'Empirisme transcendantal, PUF, 2010. Elle a dirigé le volume collectif Art et philosophie, Fontenay-aux-Roses, ENS Éditions, 1998 et publié de nombreux articles et ouvrages sur l'épistémologie de l'histoire de l'art, la question de l'image dans les domaines plastiques et cinématographiques, au croisement de la littérature, des arts non discursifs et de la philosophie. Membre du comité de rédaction des revues Chimères et Multitudes, elle dirige avec Fabienne Brugère la collection « Lignes d'art » aux PUF. 